\title{
Severe hypertriglyceridaemia responding to insulin and nicotinic acid therapy
}

\author{
STEPHEN R. SMITH \\ M.D., F.A.C.P. \\ Department of Medicine, Texas Tech. University Regional Academic Health Center, \\ El Paso, Texas 79905, U.S.A.
}

\begin{abstract}
Summary
A patient with unusually severe hypertriglyceridaemia (serum concentration initially $258 \mathrm{mmol} / \mathrm{l}$ or $22600 \mathrm{mg} /$ dl) and hypercholesterolaemia is reported and discussed. The triglyceride elevation was found to reside within the very low density lipoprotein fraction and was probably attributable to the combination of diabetes mellitus and familial hypertriglyceridaemia. Treatment with insulin and restriction of dietary carbohydrate led to a $50 \%$ reduction in the triglyceride concentration, and the addition of nicotinic acid in modest doses led ultimately to a complete normalization of the patient's lipid values. A close correlation was noted between the falling triglyceride concentration and the rising serum sodium concentration during the course of successful therapy. Overall, it is felt likely that this patient's severe and reversible hypertriglyceridaemia was on the basis of excessively rapid lipolysis leading to high concentrations of very low density lipoprotein production. Combined therapy with insulin and nicotinic acid is recommended for other patients of this nature.
\end{abstract}

\section{Introduction}

Hypertriglyceridaemia has become recognized as the commonest manifestation of hyperliproproteinaemia. Recent reports on this subject have stressed that multiple contributing factors are often present which may be of primary and/or secondary nature (Brunzell, Chait and Bierman, 1978; Brunzell and Schrott, 1973). Moreover, the existence of both primary and secondary factors is common, particularly in patients with severe degrees of hypertriglyceridaemia (Brunzell et al., 1978). Although the distinction between very low density lipoproteins (VLDL) and chylomicrons remains clear from the standpoint of in vitro recognition, the intermittent co-existence of these particles in patients with severe hypertriglyceridaemia has led to some difficulty in sharp differentiation at the clinical level (François et al., 1977). Causes for this include the fact that VLDL and chylomicrons share a common disposal mechanism and the fact that substantial heterogeneity exists within the macromolecular category defined as 'very low density lipoprotein' (Brunzell et al., 1973; Strejo, Kallai and Steiner, 1977).

The treatment of patients with severe hypertriglyceridaemia has been marked by frustration and disappointment on the part of many physicians and patients. The multiplicity of factors involved and the lack of availability of easily applied therapeutic approaches no doubt lead to this situation. In this report, a patient with unusually severe hypertriglyceridaemia, as well as hypercholesterolaemia, is presented, and his remarkably successful therapy is recounted and discussed.

\section{Case history and data}

This 54-year-old Latin-American male presented to the Emergency Room complaining of weakness, dizziness, and anorexia. The patient had a recognized history of chronic alcoholism, but it was stated by the patient and confirmed by relatives that he had not ingested alcohol during the preceding 2 weeks. There was also a history of vague abdominal discomfort of a chronic nature, and the patient had experienced melaena on several occasions during the 2 weeks before admission. He had apparently lost weight, as evidenced by looseness of his clothing.

The family history revealed an aunt with diabetes mellitus and a brother with hyperlipidaemia being treated with clofibrate.

On physical examination the patient was found to be a somewhat obese Latin male in no acute distress. Blood pressure was $150 / 100 \mathrm{mmHg}$ with a heart rate of $100 / \mathrm{min}$ and a respiratory rate of $20 / \mathrm{min}$. Examination of the skin revealed spider angiomas over the upper chest and eruptive xanthomas over the knees and heels. Examination of the fundi revealed lipaemia retinalis (Fig. 1). The conjunctivae were pale. The heart and lungs were normal. Examination of the abdomen revealed a firm liver edge 8 $\mathrm{cm}$ below the right costal margin that was nontender. The spleen was not palpable, and ascites could not be demonstrated.

Initial laboratory data included the following: 
Haematocrit was $31 \%$. WBC count was $10.5 \times 10^{9} / 1$ with $69 \%$ neutrophils, $29 \%$ lymphocytes, and $2 \%$ eosinophils. Urinalysis revealed $4+$ glucose, small acetone, and $1+$ protein. Stool occult blood was positive. The serum was grossly lipaemic. Fasting glucose was $17.0 \mathrm{mmol} / 1$ with a urea nitrogen of $0.7 \mathrm{mmol} / \mathrm{l}$. Serum sodium was $85 \mathrm{mmol} / \mathrm{l}$, and potassium was $3.4 \mathrm{mmol} / \mathrm{l}$. Serum triglyceride was initially measured at $258 \mathrm{mmol} / 1$ with cholesterol $59.8 \mathrm{mmol} / \mathrm{l}$. A heparinized sample of plasma was refrigerated for $16 \mathrm{hr}$ at $4^{\circ} \mathrm{C}$, and turbidity of the solution remained uniformly distributed at the end of this time. Serum lipoprotein electrophoresis showed $83 \%$ pre- $\beta$-, and $15 \% \beta$-, and $2 \% \alpha$-lipoprotein, along with absence of chylomicra.

An electrocardiogram was normal. Chest X-ray was normal. An abdominal X-ray was normal except for hepatic enlargement.

Insertion of a nasogastric tube confirmed a small quantity of blood in the stomach. The patient was treated with antacids and i.v. cimetidine $300 \mathrm{mg}$ 6 hourly and had no subsequent melaena. Gastric aspiration later revealed that the bleeding had stopped, and the haematocrit gradually increased during the weeks that followed.

By the second hospital day, the patient's abdominal discomfort disappeared and his appetite was much improved. He was started on an 800 calorie diet with carbohydrate limited to $35 \%$ of the caloric intake and a 4-feedings per day schedule. The fasting blood sugar on that day was $16.1 \mathrm{mmol} / \mathrm{l}$, and the patient was started on lente insulin 20 un day. During subsequent days, the patient's insudịn dose was gradually increased, and supplement regular insulin was used during the first week of the

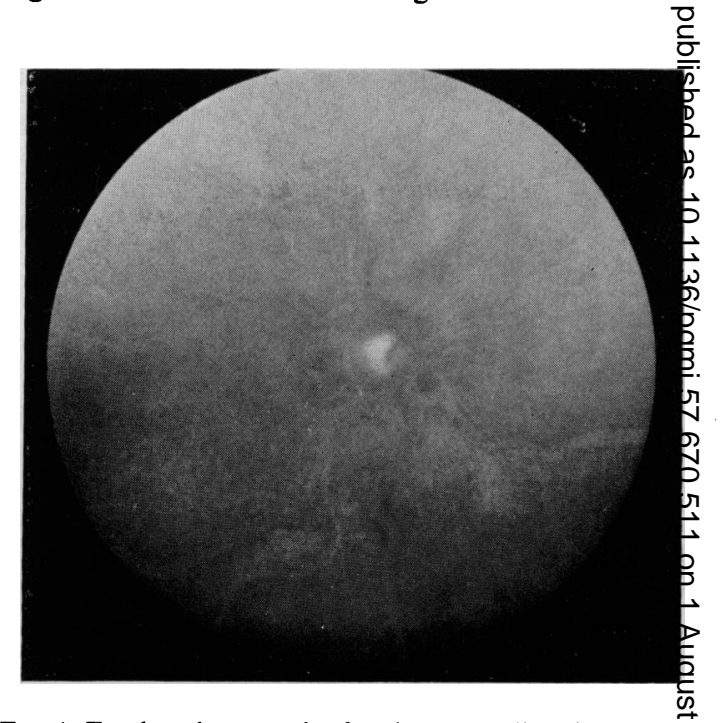

FIG. 1. Fundus photograph of patient, revealing the presenoe of lipaemia retinalis.

hospital course. Ultimately the dose was stabiß̈z at 60 units of lente insulin/day, as is indicated $\underline{\underline{m}}$ Fig. 2. There was reduction in the patient's serum

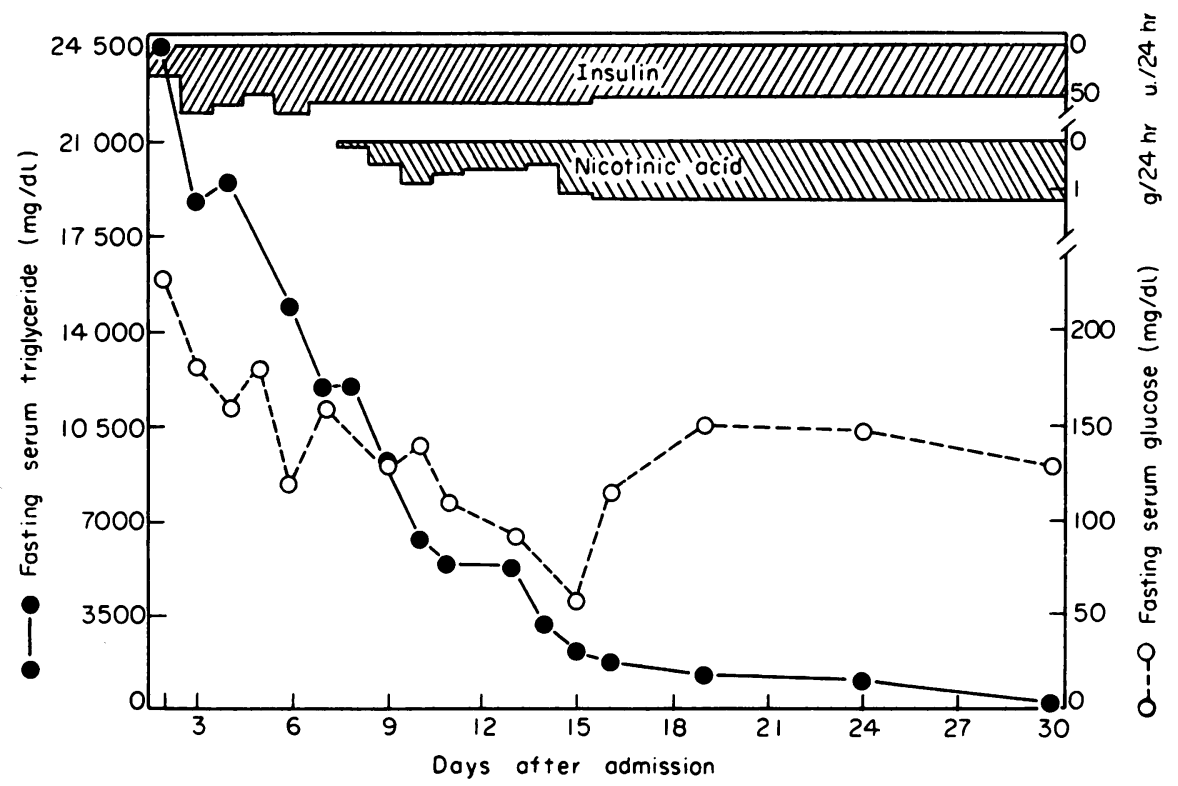

FIG. 2. Fasting serum triglyceride and glucose concentrations, initially and during treatment (based on mean triglyceride molecular weight of 875 daltons-factor 0.0114 ). 
triglyceride concentration to $132 \mathrm{mmol} / \mathrm{l}$ by the end of the first week in the hospital.

On the 9th hospital day nicotinic acid was added to the regimen, initially at a dose of $100 \mathrm{mg} 4$ times/day. Efforts were made to increase the dose within the limits of the patient's tolerance for the medication. Pruritus and skin discomfort did produce some difficulty, but ultimately the nicotinic acid was increased to $300 \mathrm{mg} 4$ times/day. The serial reduction in serum triglyceride that resulted is presented in Fig. 2. The serum cholesterol concentration fell also, ultimately reaching a level of $9.4 \mathrm{mmol} / \mathrm{l}$.

The patient was discharged home on the 17th hospital day and was instructed to continue to follow the 800 -calorie diet with $35 \%$ carbohydrate restriction. His discharge medications were lente insulin 55 units/day and nicotinic acid $300 \mathrm{mg}$ 4 times/day. The patient was observed during the subsequent month in the out-patient clinic and appeared to follow the instructions given to him at the time of discharge. His weight remained stable, his fasting glucose was in the $7 \cdot 3-8 \cdot 3 \mathrm{mmol} / 1$ range, and his serum triglyceride concentration continued to fall. At 30 days after the initial admission the patient had a serum triglyceride of $2 \cdot 1 \mathrm{mmol} / 1$ with serum cholesterol of $9.4 \mathrm{mmol} / \mathrm{l}$. His serum sodium had increased to $140 \mathrm{mmol} / \mathrm{l}$ (Fig. 3).

\section{Methods}

Serum samples were collected in the postabsorptive state and processed in a routine fashion. Serum triglyceride measurements were made by an enzymatic method for the estimation of glycerol following lipase-induced triglyceride lipolysis (Rautela et al., 1974). Most of the samples were diluted several-fold in order to produce final triglyceride concentrations of $<5.5 \mathrm{mmol} / \mathrm{l}$. Measurement of serum total cholesterol was accomplished by an enzymatic method wherein cholesterol oxidase acting upon cholesterol led to liberation of hydrogen peroxide. The hydrogen peroxide in turn led to production of an oxidized product of a chromogenic substrate, measurable by spectrophotometry (Rautela and Liedtke, 1978). A cholesterol esterase was used in the initial step in order to convert the total serum cholesterol to the free form. As in the case of the triglyceride assay, it was necessary to dilute the serum samples several-fold in order to produce a final concentration in the accurately measurable range.

The serum sample for lipoprotein electrophoresis was processed in an agarose gel electrophoresis system (Papadopoulos and Kintzios, 1971). The lipoprotein peaks were quantitated by densitometry and were expressed as percentages of the total lipoprotein material present.

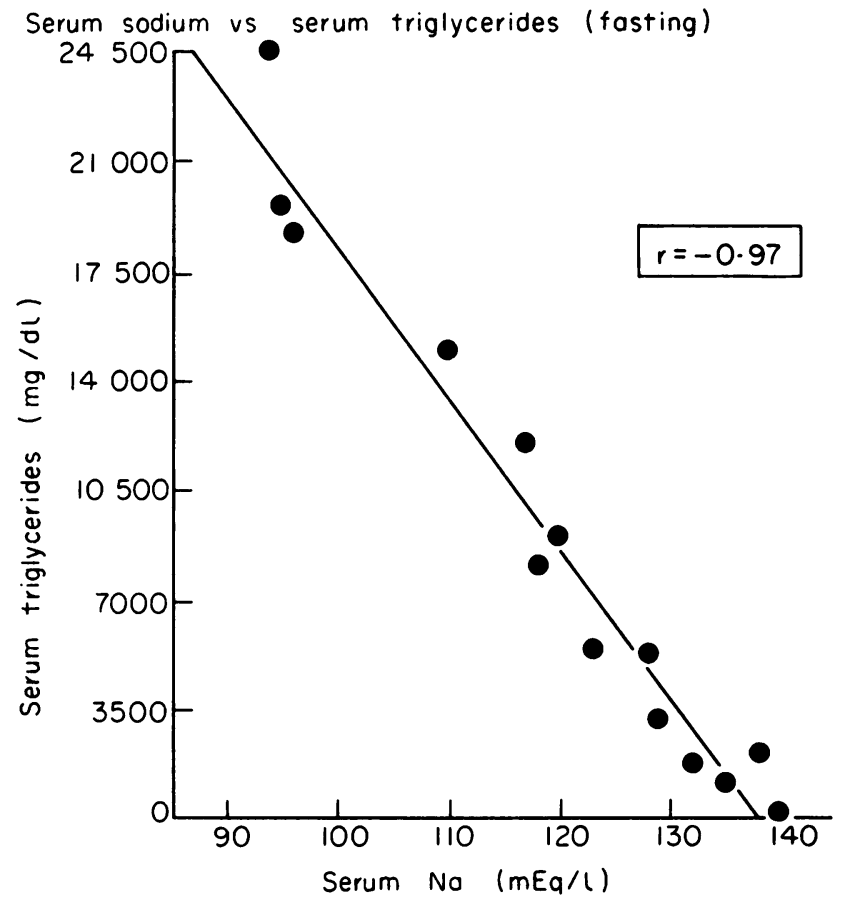

FIG. 3. Co-incident serum sodium and triglyceride concentrations obtained throughout period of treatment. (based on mean triglyceride molecular weight of 875 daltons-factor $=0.0114$ ). 


\section{Discussion}

The severity of this patient's hypertriglyceridaemie exceeds that of any reported instance found by the authors in the literature. The fasting concentration initially presented by this patient was more than twice the upper end of the range listed by Brunzell et al. (1978) in their recent discussion of marked hypertriglyceridaemia. In this case, as in others, severe hypertriglyceridaemia probably resulted from a combination of factors (Brunzell et al., 1975a; Brunzell and Schrott, 1973). Based on the absence of obvious low density lipoprotein or intermediate density lipoprotein elevation in this patient and the presence of a history of hyperlipidaemia in his brother, it appears that the underlying diagnosis would be best described as familial hypertriglyceridaemia. This condition is characterized by increased endogenous VLDL production. The occasional elevation of chylomicrons in fasting serum samples of such patients is explained on the basis of saturation of the lipoprotein lipase removal mechanism secondary to excessively high levels of VLDL production and degradation (Brunzell et al., 1973). The findings in this patient indicated a VLDL problem, with failure of the plasma to clear after $16 \mathrm{hr}$ of refrigeration and with a massive pre- $\beta$-lipoprotein peak on serum electrophoresis (Levy et al., 1972). The absence of evidence of chylomicrons in this patient's fasting samples may be construed as an indication that lipoprotein lipase was at least minimally adequate.

In addition to the primary abnormality, this patient also had secondary hyperlipoproteinaemia related to his untreated diabetes mellitus. Untreated diabetes mellitus is known to predispose patients to the development of hypertriglyceridaemia through a variety of mechanisms. The state of insulin deficiency and/or glucagon excess is associated with increased tissue lipase activity, resulting in increased transport of free fatty acids from adipose tissue to the liver (McGarry, 1979). Such fatty acids then become potential substrate for the production of VLDL. Additionally, severe insulin deficiency leads to reduction in adipose tissue lipoprotein lipase activity with the possibility that elevated serum levels of VLDL and/or chylomicrons will develop (Olefsky, Farquhar and Reaven, 1974). Finally, it is not certain that all of the evidence for accelerated VLDL synthesis in diabetes mellitus is explained on the basis of increased fatty acid flux from adipose tissue, and it may be that generous intra-hepatic insulin concentrations play a role in patients with peripheral insulin resistance due to obesity or other diabetogenic factors (Nikkila, Huttunen and Ehnholm, 1977; Kyner et al., 1976). Unfortunately, endogenous insulin concentrations were not assessed in this patient.
Strong consideration was given to the possibilit that alcoholism had contributed to the developmert and maintenance of hypertriglyceridaemia in th patient, but repeated questioning on this point to the patient and several relatives clearly indicateg that there had been no ethanol consumption for least 2 weeks. Ethanol is felt to induce intrahepat triglyceride synthesis on the basis of NAD reduction in conjunction with generous availability of acetafs (Lieber, 1977). These alterations are acute and transitory, leading to secondary hypertriglyceric aemia of only a few days' duration. On the other hand there is evidence that alcoholic liver disease leads abnormalities in lipoprotein structure and metaboa ism that can produce a more protracted hype? triglyceridaemia (Sabesin et al., 1977). Although this patient probably did have a degree of alcoholio liver disease, based on the firm, enlarged liver and the spider angiomata, it is unlikely that this mecir anism played a major role in his lipid disorder considering the absence of substantial abnormalities in liver function and the mildness of the hyper-triglyceridaemia that has been reported in this association.

Insulin therapy administered in conjunction witf the low-calorie carbohydrate-restricted diet $\$ \overrightarrow{\mathrm{ag}}$ effective in initiating a substantial reduction in patient's serum triglyceride concentration. Wi inim one week a $50 \%$ decrease had been achieved dow a level of $132 \mathrm{mmol} / \mathrm{l}$. At this point, however, the patient still had severe fasting lipaemia and it was elected to add a second agent. Insulin is likely to have improved triglyceride clearance by enhancing lipoprotein lipase activity (Brunzell, Porte ang Bierman, 1975b), and it is also probable that insulin led to a reduction in the rate of VLDL production by reducing the supply of free fatty acids to the liver (McGarry, 1979; Olefsky et al., 1974). Ad ministration of nicotinic acid in modest quantities led to a sharp further reduction in the serum trit. glyceride concentration, suggesting that this subs stance was of particular value in the treatment of the patient's hypertriglyceridaemia. Nicotinic acid is known to produce a rapid reduction in the rate of hepatic VLDL production and it is probable that this effect is largely or entirely secondary to its. potent anti-lipolytic action (Kudchodkar et abs, 1978; Yeshuran and Gotto, 1976). In a recent reviend of this subject, Levy stated that decreases in serum triglyceride level of over $60 \%$ may result from use of nicotinic acid (Levy, 1977). A maintenance dose in the range of 3 to $9 \mathrm{~g} /$ day has been recommended. In the present patient it is notable that the reduction serum triglyceride concentration ultimately achieved on nicotinic acid therapy (combined with insuli and diet restriction) was in the range of $98 \%$. Moreover, the maximum dosage used was $1.2 \mathrm{~g} / \mathrm{da}$, 
or only $40 \%$ of the minimum maintenance dose level recommended by Levy. Thus, it appears that nicotinic acid is particularly effective in the treatment of severe hypertriglyceridaemia generated by the interaction of familial hypertriglyceridaemia and untreated diabetes mellitus. Since the use of nicotinic acid is frequently limited by side effects of flushing and pruritus, the identification of an effective derivative agent lacking these effects would be of great importance. Tetranicotinoylfructose may merit an investigative trial in this regard.

The data summarized in Fig. 3 indicate a rather tight inverse correlation between the serum triglyceride and sodium concentrations. During this course of therapy the patient's serum sodium concentration could, in fact, have been used as an estimate of his triglyceride concentration, especially in the early stages of management. This relationship, if documented in several additional patients, could be used to reduce the burden on the clinical laboratory produced by repeated measurement of very high triglyceride concentrations. The relationship generated by the data in Fig. 3, if confirmed, can also be used to estimate the degree of hyponatraemia that is accounted for on the basis of a given level of hypertriglyceridaemia.

The patient reported herein presented with a level of hypertriglyceridaemia that is the highest reported in the medical literature. The aetiological factors involved were familial hypertriglyceridaemia and untreated diabetes mellitus, and the patient showed a remarkable response to treatment with diet restriction, insulin, and nicotinic acid. Considering the effectiveness of nicotinic acid in this patient and its probable major mechanism of action in hyperlipoproteinaemia, it is likely that unregulated fat turnover is the principal abnormality in patients with hypertriglyceridaemia based on this combination of aetiologies. It is therefore recommended that therapy with insulin and nicotinic acid be considered for such patients when dietary measures have proved less than entirely successful.

\section{References}

Brunzell, J.D., Chait, A. \& Bierman, E.L. (1978) Pathophysiology of lipoprotein transport. Metabolism, 27, 1109.

Brunzell, J.D., Hazzard, W.R., Motulsky, A.G. \& BiERMAN, E.L. (1975a) Evidence for diabetes mellitus and genetic forms of hypertriglyceridemia as independent entities. Metabolism, 24, 1115.

Brunzell, J.D., Hazzard, W.R., Porte Jr, D. \& Bierman, E.L. (1973) Evidence for a common, saturable, triglyceride removal mechanism for chylomicrons and very low density lipoproteins in man. Journal of Clinical Investigation, 52, 1578.

Brunzell, J.D., Porte Jr, D. \& Bierman, E.L. (1975b) Reversible abnormalities in postheparin lipolytic activity during the late phase of release in diabetes mellitus (postheparin lipolytic activity in diabetes). Metabolism, 24, 1123.

Brunzell, J.D. \& SchrotT, H.G. (1973) The interaction of familial and secondary causes of hypertriglyceridemia: role in pancreatitis. Transactions of the Association of American Physicians, 86, 245.

Francois, J., Lentini, F., Hoste, P. \& Rottiers, R. (1977) Genetic study of hyperlipoproteinemia types IV and V. Clinical Genetics, 12, 202.

Kudchodkar, B.J., SODHI, H.S., Horlick, L. \& Mason, D.T. (1978) Mechanisms of hypolipidemic action of nicotinic acid. Clinical Pharmacology and Therapeutics, 24, 354.

Kyner, J.L., LeVy, R.I., SOEldner, J.S., Gleason, R.E. \& FredRICKSON, D.S. (1976) Lipid, glucose and insulin interrelationships in normal, prediabetic, and chemical diabetic subiects. Journal of Laboratory and Clinical Medicine, 88, 345.

LEVY, R.I. (1977) The effect of hypolipidemic drugs on plasma lipoproteins. Annual Review of Pharmacology and Toxicology, 17, 499.

LeVy, R.I., Fredrickson, D.S., Shulman, R., Bilheimer, D.W., Breslow, J.L., Stone, N.J., LuX, S.E., Sloan, H.R., Krauss, R.M. \& Herbert, P.N. (1972) Dietary and drug treatment of primary hyperlipoproteinemia. Annals of Internal Medicine, 77, 267.

LIEBER, C.S. (1977) Metabolic Aspects of Alcoholism. University Park Press, Baltimore.

MCGARRY, J.D. (1979) New perspectives in the regulation of ketogenesis. Diabetes, 28, 517.

Nikkila, E.A., Hutrunen, J.K. \& Ehnholm, C. (1977) Postheparin plasma lipoprotein lipase and hepatic lipase in diabetes mellitus. Relationship to plasma triglyceride metabolism. Diabetes, 26, 11.

Olefsky, J.M., Farquhar, J.W. \& Reaven, G.M. (1974) Reappraisal of the role of insulin in hypertriglyceridemia. American Journal of Medicine, 57, 551.

Papadopoulos, N.M. \& Kintzios, J.A. (1971) Varieties of human serum lipoprotein pattern: evaluation by agarose gel electrophoresis. Clinical Chemistry, 17, 427.

Rautela, G.S., Hall Jr, R.G., Bekiesz, C.L. \& Wermus, G.R. (1974) A kinetic method for rapid and automatic measurement of triglycerides in biological fluids. Clinical Chemistry, 20, 857.

Rautela, G.S. \& LiedtKe, R.J. (1978) Automated enzymic measurement of total cholesterol in serum. Clinical Chemistry, 24, 108.

Sabesin, S.M., Hawkins, H.L., Kuiken, L. \& Ragland, J.B. (1977) Abnormal plasma lipoproteins and lecithincholesterol acyltransferase deficiency in alcoholic liver disease. Gastroenterology, 72, 510.

Strejo, D., Kallai, M.A. \& Steiner, G. (1977) The metabolic heterogeneity of human very low density lipoprotein triglyceride. Metabolism, 26, 1333.

YeshURUN, D. \& GotTo Jr, A.M. (1976) Drug treatment of hyperlipidemia. American Journal of Medicine, 60, 379. 